\title{
Complete cytogenetic response to Nilotinib in a chronic myeloid leukemia case with a rare e13a3(b2a3) BCR-ABL fusion transcript: A case report
}

\author{
BEI LIU $^{1 *}$, WEI ZHANG ${ }^{2 *}$ and HAIZHEN MA ${ }^{1}$ \\ ${ }^{1}$ Department of Hematology; ${ }^{2}$ Central Laboratory, The First Affiliated Hospital, \\ Lanzhou University, Lanzhou, Gansu 730000, P.R. China
}

Received March 8, 2015; Accepted December 11, 2015

DOI: $10.3892 / \mathrm{mmr} .2016 .4826$

\begin{abstract}
In the present study, an atypical case of chronic myeloid leukemia (CML) in a 32-year-old male was reported. CML cases with e13a3 breakpoint cluster region (BCR)-ABL transcripts are extremely rare. Reverse transcription quantitative-polymerase chain reaction (RT-qPCR) was initially negative due to the primer corresponding to $\mathrm{ABL}$ a2 sequences and diagnosis was based upon analysis of the bone marrow smear, fluorescence in situ hybridization and karyotype analysis. RT-qPCR analysis with the ABL primer, which was located in ABL exon 3 to enable the detection of fusions with either ABL a2 or exon a 3 demonstrated the presence of the BCR-ABL fusion transcript e13a3. The patient responded well to Nilotinib and achieved a complete cytogenetic response after 3 months.
\end{abstract}

\section{Introduction}

CML is a malignant clonal disorder of pluripotent hematopoietic stem cells. Patients with CML have a t $(9 ; 22)(\mathrm{q} 34 ; \mathrm{q} 11)$ translocation that results in a breakpoint cluster region (BCR)-ABL fusion gene. In general, three breakpoint cluster regions in the BCR gene have been described: Major (M-bcr), minor (m-bcr) and micro (u-bcr). The M-bcr region consists of BCR introns downstream of either exon 13 (e13, previously known as b2) or 14 (e14, previously known as b3) and introns upstream of ABL exon 2 (a2). These BCR-ABL e13a2 and e14a2 fusions result in a $210-\mathrm{kDa}$ fusion protein. $\mathrm{m}-\mathrm{bcr}$ and $\mathrm{u}$-bcr are two less common breakpoints in the intronic region between the alternative BCR exon 2 and exons 19 and 20,

Correspondence to: Professor Haizhen Ma, Department of Hematology, The First Affiliated Hospital, Lanzhou University, 1 Donggangxilu Street, Lanzhou, Gansu 730000, P.R. China

E-mail: mahaizhen-2008@163.com

${ }^{*}$ Contributed equally

Key words: chronic myeloid leukemia, e13a3, Nilotinib which encode a 190-kDa (e1a2) and 230-kDa fusion protein (e19a2), respectively.

However, a number of 'atypical' BCR-ABL transcripts (e1a3, e13a3, e14a3, e19a3, e6a2 and e8a2) resulting from chromosomal breakpoints outside the ABL intron 1 or BCR intron 1,13 or 14 , have been reported (1). These atypical transcripts may escape detection when using methods that are optimized to detect only the typical ones (1).

In the present study, a case of CML, which tested positive for the BCR-ABL translocation by fluorescence in situ hybridization (FISH) and cytogenetic analysis, but tested negative by reverse transcription quantitative-polymerase chain reaction (RT-qPCR) molecular analysis at the time of diagnosis was reported. Further RT-qPCR analysis with alternative primer sets demonstrated the presence of an e13a3 BCR-ABL fusion gene (2), in which ABL exon 3 rather than exon 2 was fused to $\mathrm{BCR}$, which is extremely rare (3). BCR-ABL with the e13a3 transcript in CML patients, however, usually predicts improved treatment response and a longer survival time (4). This patient responded immediately to Nilotinib with the achievement of a complete cytogenetic remission.

\section{Case report}

A 32-year-old male was admitted to the Department of General Surgery at The First Affiliated Hospital of Lanzhou University (Lanzhou, China) in June 2014 with a history of weight loss and splenomegaly. Routine peripheral blood analysis demonstrated white blood cell (WBC) counts of $310.10 \times 10^{9} / 1$ ( $2 \%$ blasts, $2 \%$ promyelocytes, $25 \%$ myelocytes, $9 \%$ metamyelocytes, $19 \%$ band neutrophils, $26 \%$ segmented neutrophils, $5.0 \%$ basophils, $9 \%$ eosinophils and $3 \%$ lymphocytes), a hemoglobin count of $8.7 \mathrm{~g} / \mathrm{dl}$ and a platelet count of $130 \times 10^{9} / 1$. In conclusion, the patient was diagnosed with leukemia and admitted to the Department of Hematology. Following this, the patient underwent a bone marrow aspirate, which demonstrated hypercellularity with a marked myeloid predominance. Bone marrow mononuclear cells were cultured according to standard methods and the karyotypes were analyzed by G-banding, which demonstrated 46,XY, t $(9,22)$ in 20/20 metaphases (Fig. 1). The positive 


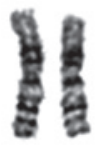

1

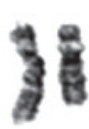

6

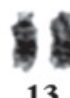

13

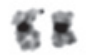

19

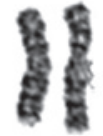

2

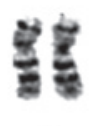

7
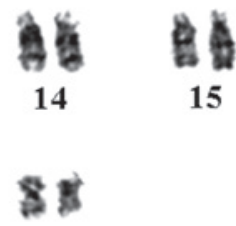

20

3

8
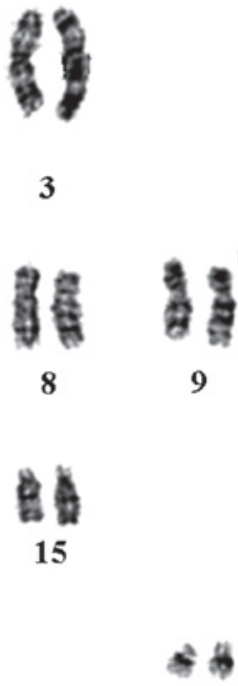

21

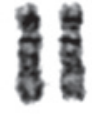

is

4

5


10

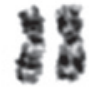

11

12



Figure 1. G-banded karyotype. Arrows indicate the $\mathrm{t}(9 ; 22)$ translocation.



Figure 2. FISH analysis showing a typical pattern of $\mathrm{t}(9 ; 22)$ (1O1G2R). FISH was performed using a GLP BCR/GLP ABL to identify BCR-ABL fusion genes. A normal cell shows two separate sets of red and green signals $(2 \mathrm{R} 2 \mathrm{G})$ while a cell containing a reciprocal $\mathrm{t}(9 ; 22)$ translocation shows individual red and green signals from the normal 9 and 22 chromosomes and red/green fusion signals from the derivative 9 and 22 chromosomes (1O1G2R). The two fusions are indicated by arrows. FISH, fluorescence in situ hybridization; GLP, gene locus-specific probe.

rate of BCR-ABL fusion was $100 \%$ as determined by FISH (results were considered clonal when the percentage of cells containing a reciprocal $\mathrm{t}(9 ; 22)$ exceeded $4.0 \%$; Fig. 2$)$. No BCR-ABL fusion gene (e13a2, e14a2, e1a2 and e19a2) was detected by RT-qPCR. However, further detection by RT-PCR demonstrated the presence of the e13a3 fusion gene (Fig. 3). Subsequently, the patient was treated initially with hydroxyurea $(3,000 \mathrm{mg} /$ day) with reasonably good control of WBCs. After 3 weeks, the WBC count was decreased $\left(9.8 \times 10^{9} / 1\right)$ and the previous relevant symptoms disappeared. Following this, the patient was administered $800 \mathrm{mg}$ of Nilotinib daily. No side effects or hematologic toxicity were observed. The karyotype was normalized [46,XY (20/20)] and FISH for BCR-ABL decreased to $0 \%$ after 3 months on Nilotinib, indicating a complete cytogenetic response (Fig. 4). Written informed consent was obtained from the patient and the study was approved by the Ethics Committee of The First Hospital of Lanzhou University.

\section{Discussion}

BCR-ABL transcripts with intronic breakpoints downstream of $\mathrm{ABL}$ a2, lacking $\mathrm{ABL}$ exon 2, are rare. The present study described a chronic myeloid leukemia (CML) case with an e13a3 BCR-ABL fusion transcript. To date, only 16 cases of CML with e13a3 BCR-ABL transcripts have been reported (3,5-7). Ito et al reported the frequency and distribution of BCR-ABL transcript types among the Japanese. Overall, the percentage of patients with the e14a2, e13a2 and e13a 3 transcript types was 67.50 (85/126), 30.20 (38/126) and $0.80 \%$ (1/126), respectively (8). Goh et al reported that the majority of patients $(538 / 548,98.18 \%)$ were found to have e $14 \mathrm{a} 2$ or e13a2 in Korea, and the frequency of occurrence of e13a3 was $0.18 \%$ (1/548) (9). In a previous study by Todoric-Zivanovic et al, the e14a2 form of BCR-ABL was detected in 100 patients (73.5\%) and the e13a2 form was detected in 34 patients (25\%). One (0.75\%) patient had the ela1 transcript of BCR-ABL, however, no BCR-a3 case was detected (10).

The number of reported BCR-a3 cases is small compared with the theoretical frequency of BCR-a3 cases. An explanation for this mismatch may be due to the methodology of RT-qPCR. Initially, the e13a3 fusion transcript was missed by RT-qPCR using the primer corresponding to $\mathrm{ABL}$ a2 sequences despite the existence of the $\mathrm{t}(9 ; 22)(\mathrm{q} 34 ; \mathrm{q} 11)$ translocation by G-banding. In addition, FISH also detected this translocation due to the large size of the probes used. Following this, RT-qPCR analysis with the ABL primer, which was located in ABL exon 3 to enable the detection of fusions with either ABL exon 2 (a2) or exon 3 (a3) demonstrated a $169 \mathrm{bp}$ band in the present case, in comparison with an e13a2-positive control band ( $343 \mathrm{bp}$ ), suggesting that the $\mathrm{ABL}$ a 2 region (174 bp) was completely deficient. There may be more cases that present BCR-a3 fusion transcripts if a proper 


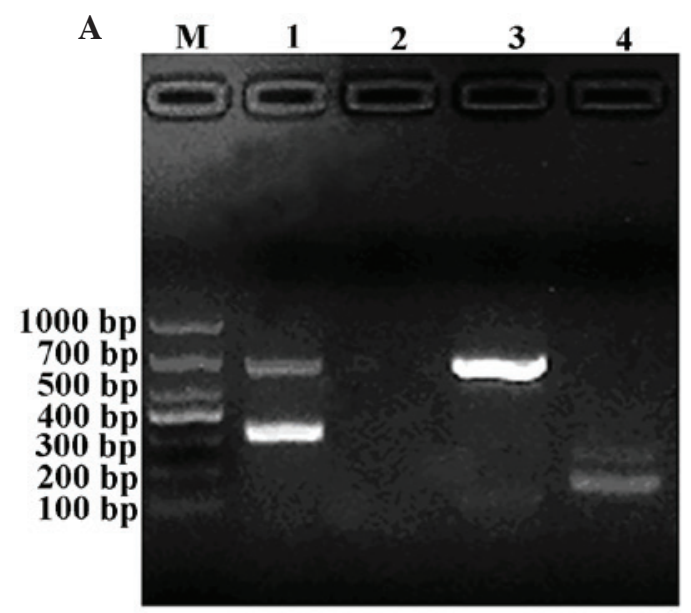

B

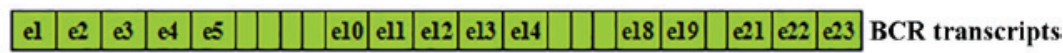
\begin{tabular}{|l|l|l|l|l|l|l|l|l|l|l|}
\hline al & 22 & 23 & a4 & 25 & 26 & a7 & a8 & a9 & al0 & all \\
\hline
\end{tabular} ABL transcripts

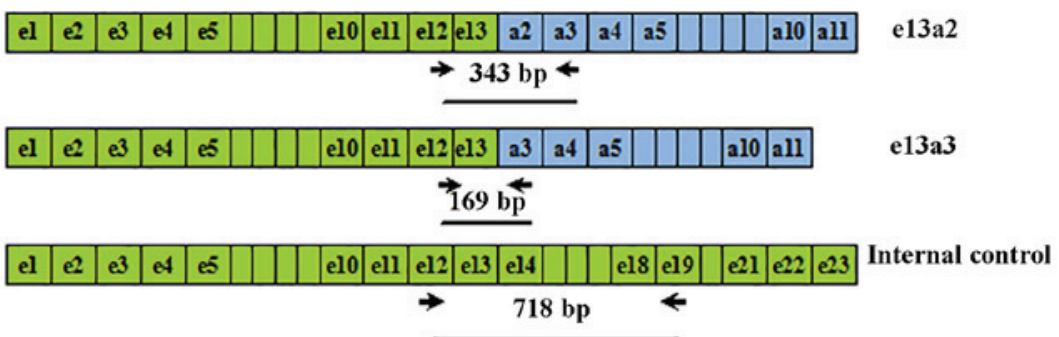

Figure 3. Polymerase chain reaction products and schematic representation of BCR-ABL transcripts. (A) Lane M, bp markers; lane 1, e13a2-positive control (343 bp); lane 2, negative control; lane 3, internal control (718 bp); lane 4, patient with e13a3 (169 bp). (B) The arrows indicate the position of the primers. The ABL primer was located in ABL exon 3 to enable the detection of fusions with either ABL exon 2 (a2) or exon 3 (a3) (5'-CCATTGTGATTATAGCCTAAGACC CGGAG-3'). The BCR primer was located in exon 12 (e12) for the M-bcr transcripts (5'-AGAACATCCGGGAGCAGCAGAAGAA-3'). The internal control primers were 5'-AGAACATCCGGGAGCAGCAGAAGAA-3' and 5'-ATGTCCGTGGCCACACCGGACAC-3', which were from the normal BCR gene (1).

A


B



Figure 4. Follow up of G-banded and FISH analysis. (A) G-banding demonstrated that the patient's karyotype was altered from the t(9;22) translocation to a normal karyotype following treatment with Nilotinib for 3 months. (B) FISH demonstrated a markedly reduced BCR-ABL fusion rate of $0 \%$ (2G2R), indicating a complete cytogenetic response. FISH, fluorescence in situ hybridization.

primer is used routinely in RT-qPCR. The patient was administered $800 \mathrm{mg}$ of Nilotinib daily, and using classic cytogenetics, the $\mathrm{Ph}^{+}$metaphases decreased from $100 \%$ prior to Nilotinib treatment to $0 \%$ by 3 months. The patient did respond quickly and completely to Nilotinib, with rapid achievement of complete hematologic and cytogenetic remission.

The ABL a2 region encodes a part of the Src homology (SH)3 domain. The SH3 domain is considered to have a negative regulatory role in the kinase domain (SH1). Therefore, the lack of a SH3 domain may result in a more aggressive form of Ph-positive leukemia. By contrast, the SH3 domain is required for activation of signal transducer and activator of transcription 5 by the BCR-ABL protein, leading to full leukemogenesis. Thus, deletion of the SH3 domain may induce a less progressive clinical course $(5,11)$. The BCR-ABL a3 breakpoint does not alter the sequence coding for the ATP/imatinib binding domain, but alterations in tertiary structure compared with a typical a2 fusion could affect drug response. The clinical outcomes specific to CML patients with BCR-ABL a3 fusions are difficult to define due to the limited number of cases reported $(2,4)$.

In conclusion, CML with a BCR-ABL a3 fusion gene is a rare and challenging disease, which could lead to negative RT-qPCR results and be erroneously interpreted. According to National Comprehensive Cancer Network $(\mathrm{NCCN})$ practice guidelines, cytogenetics, FISH and RT-qPCR are recommended as the initial workup for chronic phase adult CML, as each essay can provide unique information. As mentioned, 
FISH and standard cytogenetics can identify uncommon BCR-ABL translocations that may be missed by RT-qPCR. Karyotyping identifies other cytogenetic abnormalities that may have prognostic significance. Once a complete cytogenetic remission has been obtained, the NCCN guidelines recommend RT-qPCR every 3 to 6 months, which may not be effective for patients with rare breakpoints. Further studies are required to interpret natural frequency and unique clinical manifestations of this rare BCR-ABL fusion in patients with CML.

\section{Acknowledgements}

The authors would like to thank High Trust Diagnostics Inc. and Dr Ren Li and Dr Luo Xiu Feng for their assistance with the experiment.

\section{References}

1. Burmeister T and Reinhardt R: A multiplex PCR for improved detection of typical and atypical BCR-ABL fusion transcripts. Leuk Res 32: 579-585, 2008

2. Jinawath N, Norris-Kirby A, Smith BD, Gocke CD, Batista DA, Griffin CA and Murphy KM: A rare e14a3 (b3a3) BCR-ABL fusion transcript in chronic myeloid leukemia: Diagnostic challenges in clinical laboratory practice. J Mol Diagn 11: 359-363, 2009.

3. Achkar WA, Wafa A, Ali BY, Manvelyan M and Liehr T: A rare chronic myeloid leukemia case with Philadelphia chromosome, BCR-ABL e13a3 transcript and complex translocation involving four different chromosomes. Oncol Lett 1: 797-800, 2010.
4. Pienkowska-Grela B, Woroniecka R, Solarska I, Kos K, Pastwińska A, Konopka L and Majewski M: Complete cytogenetic and molecular response after imatinib treatment for chronic myeloid leukemia in a patient with atypical karyotype and BCR-ABL b2a3 transcript. Cancer Genet Cytogenet 174: 111-115, 2007.

5. Fujisawa S, Nakamura S, Naito K, Kobayashi M and Ohnishi K: A variant transcript, ela3, of the minor BCR-ABL fusion gene in acute lymphoblastic leukemia: Case report and review of the literature. Int J Hematol 87: 184-188, 2008.

6. Masuko M, Furukawa T, Abe T, Wada R, Maruyama S, Kitajima T, Shibasaki Y, Toba K, Okada M and Aizawa Y: A chronic myeloid leukemia patient with atypical karyotype and BCR-ABL e13a3 transcript caused by complex chromosome rearrangement. Int J Hematol 90: 230-234, 2009.

7. De Braekeleer E, Douet-Guilbert N, Rowe D, Bown N, Morel F, Berthou C, Férec C and De Braekeleer M: ABL1 fusion genes in hematological malignancies: A review. Eur J Haematol 86: 361-371, 2011.

8. Ito T, Tanaka H, Tanaka K, Ito K, Kyo T, Dohy H, Kamada N and Kimura A: Insertion of a genomic fragment of chromosome 19 between BCR intron 19 and ABL intron 1a in a chronic myeloid leukaemia patient with micro-BCR-ABL (e19a2) transcript. $\mathrm{Br}$ J Haematol 126: 752-753, 2004

9. Goh HG, Hwang JY, Kim SH, Lee YH, Kim YL and Kim DW: Comprehensive analysis of BCR-ABL transcript types in Korean CML patients using a newly developed multiplex RT-PCR. Transl Res 148: 249-256, 2006.

10. Todoric-Zivanovic B, Strnad M, Stamatovic D, Tukic L, Krtolica K, Tatomirovic Z, Djordjevic V, Bogdanovic A, Jankovic G and Magic Z: Frequency of BCR-ABL fusion transcripts in Serbian patients with chronic myeloid leukemia. J BUON 16: 104-107, 2011.

11. Snyder DS, McMahon R, Cohen SR and Slovak ML: Chronic myeloid leukemia with an e13a3 BCR-ABL fusion: Benign course responsive to imatinib with an RT-PCR Advisory. Am J Hematol 75: 92-95, 2004. 